\title{
Synthesis of new cyclopentane phosphine oxides
}

\author{
Pelayo Camps,* Gisela Colet, Sergi Segura, and Santiago Vázquez* \\ Laboratori de Química Farmacèutica (Unitat Associada al CSIC), Facultat de Farmàcia, \\ Universitat de Barcelona, Av. Diagonal 643, E-08028, Barcelona, Spain \\ E-mail: camps@ub.edu,svazquez@ub.edu
}

\section{Dedicated to Prof. Dr. Joan Bosch on his $60^{\text {th }}$ anniversary}

\begin{abstract}
(1r,2t,5t)-2-Diphenylphosphinoyl-5-[(diphenylphosphinoyl)methyl]cyclopentanol

(10) was prepared in good yield from 3-methoxy-2-cyclopentenone by a five-step sequence which takes advantage of the electrophilic character of positions 1 and 3 of the starting material.
\end{abstract}

Keywords: Phosphine oxides, stereoselective epoxidation, Caglioti reaction, DFT calculations, GIAO, nucleophilic epoxide opening

\section{Introduction}

As part of our current interest in the synthesis of carbocyclic analogs of cis-MCCPM, ${ }^{1}$ we have described the synthesis of bisphosphinoyl compounds 1a-c, in racemic form and a straightforward diastereoselective synthesis of diphosphines 2a-b (Figure 1). ${ }^{2}$ However, several attempts to study the catalytic activity in hydrogenation reactions of metal complexes derived from diphosphines $\mathbf{2 a}$ and $\mathbf{2} \mathbf{b}$ were unsuccessful.

Taking into account these results, we considered the preparation of diphosphines of general structure I, with a free or substituted hydroxyl group, to study the catalytic activity of different metal complexes derived from them in hydrogenation reactions. Herein we describe an unsuccessful approach to this kind of diphosphine that has led to a trans-1,3-disubstituted cyclopentane 1,4-diphosphine dioxide, via new cyclopentane phosphine oxides. 


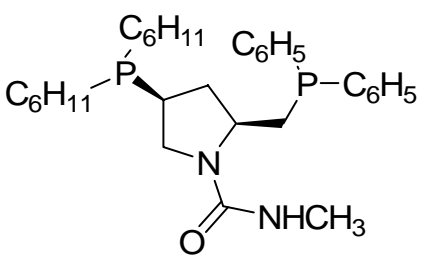

(S,S)-cis-MCCPM<smiles>[R5]CP(C)(=O)C[C@@H]1CC([PH]([R])=O)C[C@@H]1O</smiles>

1a, $\mathrm{R}=\mathrm{C}_{6} \mathrm{H}_{5}$

1b, $\mathrm{R}=$ Cyclopentyl

1c, $\mathrm{R}=\mathrm{isoPr}$<smiles>[R]P([R])C1CCC(CP(C)(C)(C)C)C1</smiles>

2a, $\mathrm{R}=\mathrm{C}_{6} \mathrm{H}_{5}$ 2b, R=Cyclopentyl<smiles>[R]P([R])[C@H]1CC[C@@H](CP(C)(C)(C)C)[C@@H]1O</smiles>

I

Figure 1

\section{Results and Discussion}

Our proposed synthesis of diphosphines of general structure I hinged on the well-known ringopening reaction of epoxides by the anion of disubstituted phosphines. The required trans epoxide, 3 , could be obtained, in racemic form, through stereoselective epoxidation of an alkene itself arising from the reduction of tosylhydrazone 5, easily obtainable from the known enone 6.

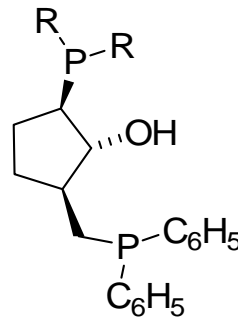

I<smiles>CCCCCP(=O)(Cc1ccccc1)CC1CC=CC1</smiles>

4<smiles>[R]P([R])(=O)C1CCC(CP(=O)(O)CCCC)[C@@H]1O</smiles>

II

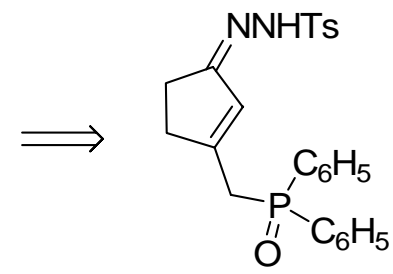

5<smiles>C=CCC1CCC2OC12CP(=O)(CCCC)c1ccccc1</smiles>

3

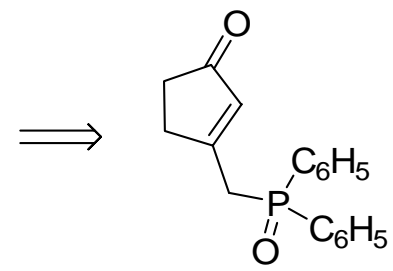

6

\section{Scheme 1}

Compound 6 was first described in 1991, following a two-step sequence from 2cyclopentenone through 1,2-nucleophilic addition of the lithium derivative of methyldiphenylphoshine oxide followed by $\mathrm{CrO}_{3}$-mediated oxidation of the intermediate allylic alcohol. ${ }^{3}$ We have found that $\mathbf{6}$ can be synthesized in a one-step process by direct addition of the lithium derivative of methyldiphenylphoshine oxide to commercially available 3-methoxy-2- 
cyclopentenone, 7 , in $43 \%$ yield. Although the overall yields of both approaches are very similar, the new one does not involve purification of any intermediate by column chromatography, so it is more convenient for the large scale preparation of $\mathbf{6}$. Reaction of enone $\mathbf{6}$ with tosylhydrazide led to the corresponding hydrazone, 5 , in $75 \%$ yield.

It is well-known that reduction of tosylhydrazones to hydrocarbons with hydride reagents such as $\mathrm{LiAlH}_{4}, \mathrm{NaB}(\mathrm{CN}) \mathrm{BH}_{3}$, or $\mathrm{NaBH}_{4}$ in acidic medium ("Caglioti reaction") is a mild alternative to the classical Wolff-Kishner and Clemmensen procedures. ${ }^{4}$ In $\alpha, \beta$-unsaturated derivatives, the carbon-carbon double bond migrates to the original carbonyl carbon atom. ${ }^{5}$ In our hands, reduction of 5 with $\mathrm{NaBH}_{4}$ in acetic acid at $70{ }^{\circ} \mathrm{C}$ led to 4 in $60 \%$ isolated yield.

The epoxidation of $\mathbf{4}$ with $\mathrm{m}$-CPBA in dichloromethane led to a mixture of two stereoisomeric epoxides in the ratio of $7: 1{ }^{6}$ Column chromatography allowed us to separate both stereoisomers in $61 \%$ and $9 \%$ yield, respectively. Both stereoisomers were fully characterized by spectroscopic techniques and elemental analyses. The ${ }^{1} \mathrm{H}$ and ${ }^{13} \mathrm{C}$ NMR spectra of these epoxides could be fully assigned on the basis of ${ }^{1} \mathrm{H} /{ }^{1} \mathrm{H}$ COSY and ${ }^{1} \mathrm{H} /{ }^{13} \mathrm{C}$ HETCOR experiments. Moreover, most of the ${ }^{1} \mathrm{H} /{ }^{1} \mathrm{H}$ coupling constants were measured, from which values the relative configuration and preferential conformation for each epoxide was proposed. Table 1 collects the experimental ${ }^{1} \mathrm{H} /{ }^{1} \mathrm{H}$ coupling constants for each epoxide and the preferred conformation suggested for these epoxides. In both cases, an envelope conformation with the $\mathrm{C} 4$ carbon atom on the same side of the oxygen oxirane atom seems to be the preferred one, no matter if the diphenylphosphinoylmethyl group occupies a pseudoequatorial (8) or a pseudoaxial (3) arrangement. Contrary to our expectations, the main epoxide, 8, has the diphenylphosphinoylmethyl group in a cis arrangement with respect to the epoxide function.

Oxidation of 4 with excess of dimethyldioxirane in acetone ${ }^{7}$ gave a mixture of both epoxides 8 and 3 in the ratio of 5:1, in $83 \%$ yield. Thus, both epoxidation procedures show similar stereoselectivity in favor of epoxide $\mathbf{8}$. 
<smiles>COC1=CC(=O)CC1</smiles>

7

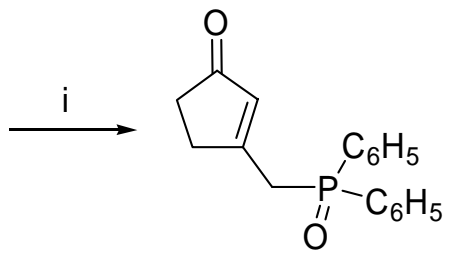

6

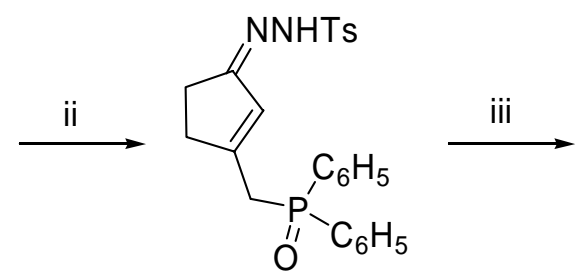

5

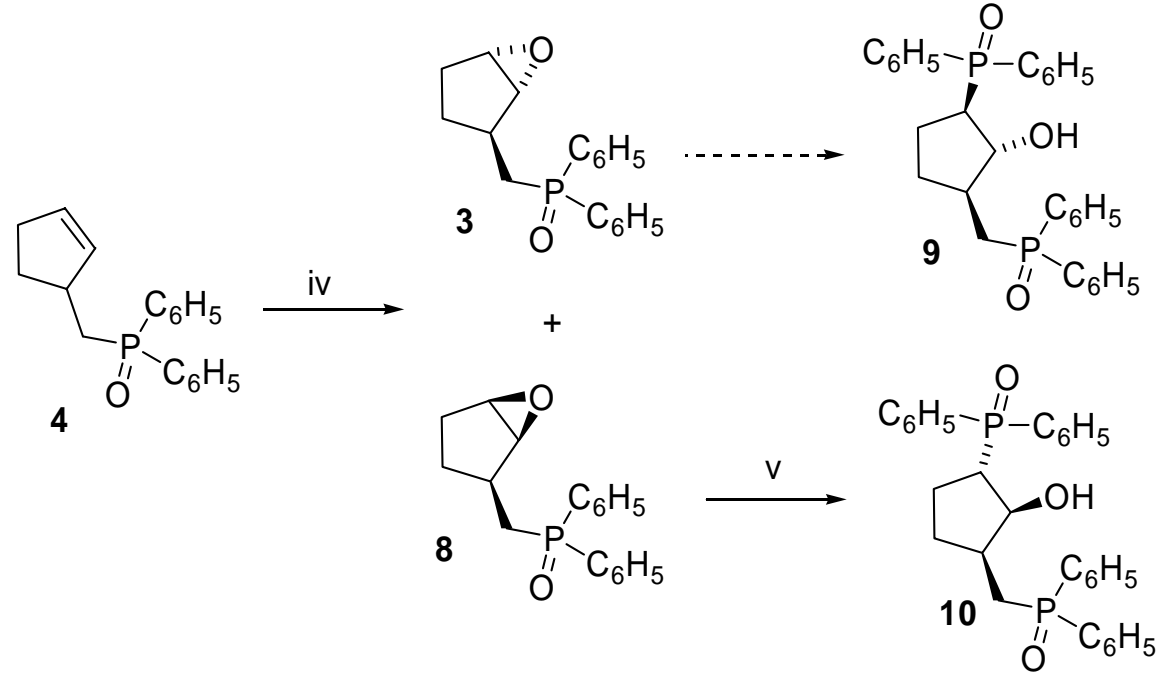

Scheme 2. Reagents and conditions: (i) Methyldiphenylphosphine oxide, $n$-BuLi, THF, $-20{ }^{\circ} \mathrm{C}$; then $10 \%$ aqueous $\mathrm{H}_{2} \mathrm{SO}_{4},-5{ }^{\circ} \mathrm{C}, 30 \mathrm{~min}$; (ii) Tosylhydrazide, acetic acid, rt, overnight; (iii) $\mathrm{NaBH}_{4}$, acetic acid, rt, $1 \mathrm{~h}$, then reflux, $3 \mathrm{~h}$; (iv) $m-\mathrm{CPBA}, \mathrm{CH}_{2} \mathrm{Cl}_{2}, \mathrm{rt}, 18 \mathrm{~h}$; (v) $\mathrm{ClP}\left(\mathrm{C}_{6} \mathrm{H}_{5}\right)_{2}$, molten sodium, 1,4-dioxane, reflux, $3 \mathrm{~h}$; then add $\mathbf{8},-30{ }^{\circ} \mathrm{C}$, overnight; then $30 \%$ aqueous $\mathrm{H}_{2} \mathrm{O}_{2}$.

DFT theoretical calculations (see below for details) carried out on the model dimethylphosphinoylmethyl epoxides, 11 and 12, showed the preferred conformation of these epoxides, to be the same deduced for epoxides 8 and $\mathbf{3}$ on the basis of their ${ }^{1} \mathrm{H} /{ }^{1} \mathrm{H}$ coupling constants measured on the ${ }^{1} \mathrm{H}$ NMR spectra (Figure 2).
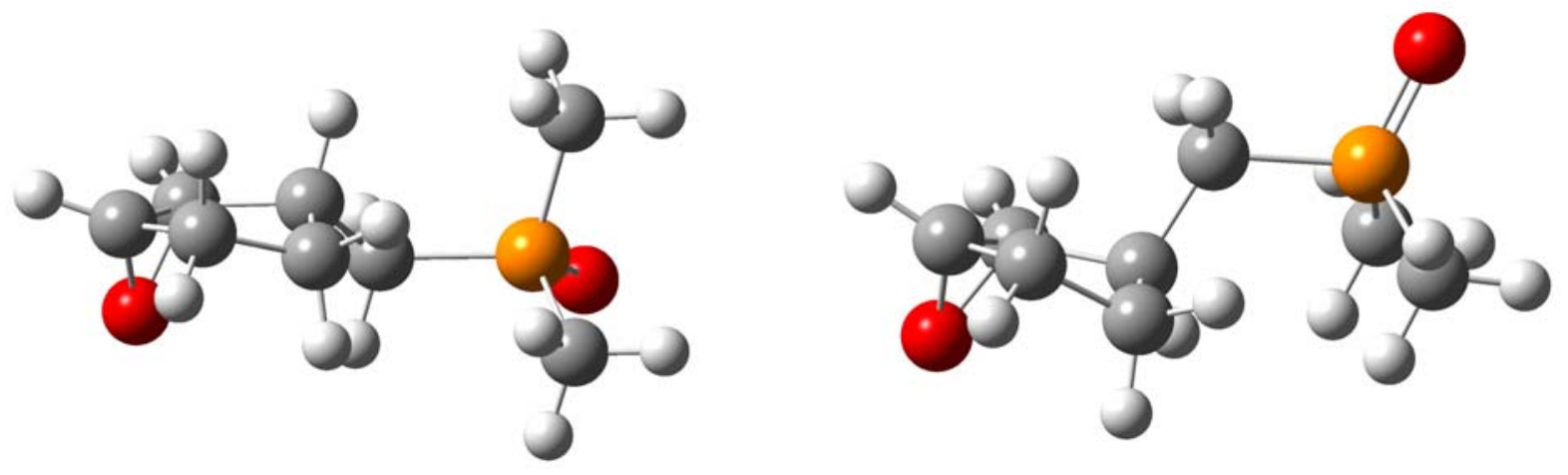

Figure 2. Calculated minimum energy conformations for the dimethylphosphinoyl compounds 11 (left side) and 12 (right side), model analogs of epoxides 8 and 3, respectively. 
Table 1. Experimental intra-annular ${ }^{1} \mathrm{H} /{ }^{1} \mathrm{H}$ coupling constants $(\mathrm{Hz})$ of epoxides 8 and $\mathbf{3}$ and corresponding calculated values for the model epoxides $\mathbf{1 1}$ and $\mathbf{1 2 .}$

\begin{tabular}{|c|c|c|c|c|}
\hline & 管 & 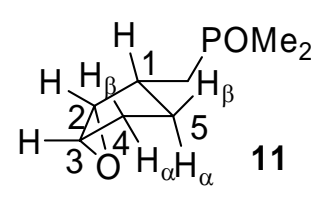 & 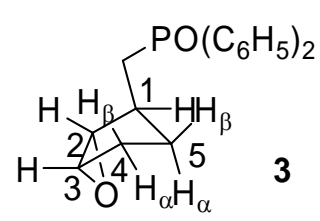 & 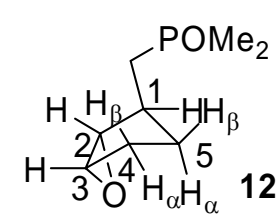 \\
\hline $\begin{array}{l}\text { Coupling } \\
\text { constant }\end{array}$ & Experimental & Calculated & Experimental & Calculated \\
\hline${ }^{3} J_{1-\mathrm{H} / 2-\mathrm{H}}$ & 2.5 & 1.5 & Very small & 0.7 \\
\hline${ }^{3} J_{1-\mathrm{H} / 5-\mathrm{H} \alpha}$ & 10.0 & 8.2 & 7.0 & 7.4 \\
\hline${ }^{3} J_{1-\mathrm{H} / 5-\mathrm{H} \beta}$ & 8.5 & 7.4 & Very small & 2.4 \\
\hline${ }^{3} J_{2-\mathrm{H} / 3-\mathrm{H}}$ & Very small & 2.2 & 2.0 & 2.2 \\
\hline${ }^{3} J_{3-\mathrm{H} / 4-\mathrm{H} \beta}$ & 2.0 & 1.5 & 1.0 & 1.3 \\
\hline${ }^{2} J_{4-\mathrm{H} \alpha / 4-\mathrm{H} \beta}$ & 14.0 & 14.8 & 14.0 & 14.8 \\
\hline${ }^{3} J_{4-\mathrm{H} \alpha / 5-\mathrm{H} \alpha}$ & 8.0 & 7.8 & 7.5 & 7.9 \\
\hline${ }^{3} J_{4-\mathrm{H} \beta / 5-\mathrm{H} \alpha}$ & 10.0 & 8.8 & 10.0 & 9.0 \\
\hline${ }^{3} J_{4-\mathrm{H} / 5-\mathrm{H} \beta}$ & 8.5 & 7.9 & 9.0 & 8.0 \\
\hline${ }^{2} J_{5-\mathrm{H} \alpha / 5-\mathrm{H} \beta}$ & 12.5 & 13.4 & 13.0 & 13.9 \\
\hline
\end{tabular}

Although the dialkylphosphine anion-induced opening of epoxides is a well precedented reaction, ${ }^{8}$ several attempts to react epoxide 8 with the lithium anion of dicyclohexylphosphine or the sodium anion of diisopropylphosphine led only to the recovery of the starting epoxide. However, when 8 was treated with an excess of the sodium salt of diphenylphosphine ${ }^{9}$ and after an oxidative work-up, the new diphosphine dioxide $\mathbf{1 0}$ was isolated in $80 \%$ yield, which was fully characterized on the basis of its spectroscopic data and elemental analysis. As expected, the attack of the diphenylphosphinyl group had only taken place at C3. Calculations on the model compound 13, having methyl instead of phenyl groups, give the envelope conformation shown in Figure 3 as the preferred one. In this conformation the $\mathrm{C} 4$ is out the plane, while the rest of the annular carbon atoms lie essentially in the same plane, with all of the substituents in pseudoequatorial positions. Comparison of the few experimental intraannular ${ }^{1} \mathrm{H} /{ }^{1} \mathrm{H}$ coupling constant values $(\mathrm{Hz})$ that could be measured from the ${ }^{1} \mathrm{H}$ NMR spectrum of alcohol $\mathbf{1 0}$ with those calculated for the model compound $\mathbf{1 3}$ (in parenthesis) showed a good agreement: ${ }^{3} J_{1-\mathrm{H} / 2 \mathrm{H}}$ $=3.0 \mathrm{~Hz}(1.3) ;{ }^{3} J_{1-\mathrm{H} / 5-\mathrm{H}}=6.0(6.6) ;{ }^{3} J_{2-\mathrm{H} / 3-\mathrm{H} \beta}=9.0 \mathrm{~Hz}(9.1) ;{ }^{3} J_{2-\mathrm{H} / 3-\mathrm{H \alpha}}=9.0(8.5)$. 


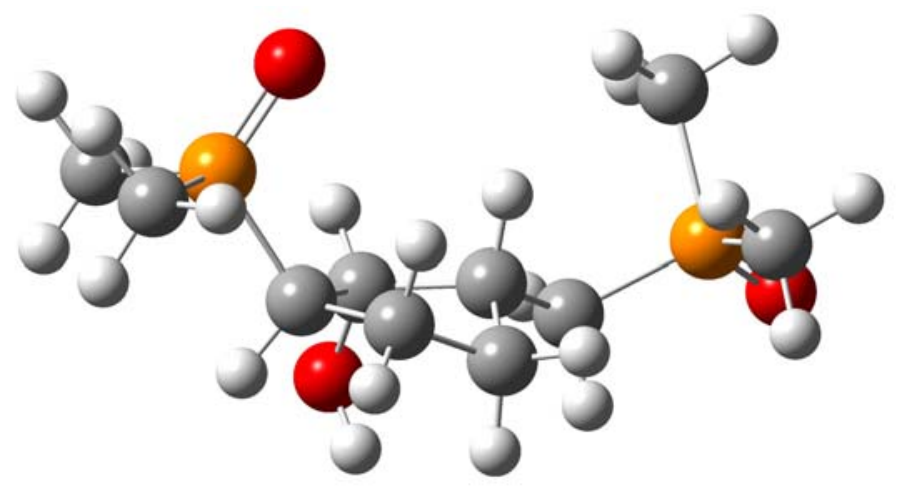

Figure 3. Calculated minimum energy conformation for compound 13, a model analog of $\mathbf{1 0 .}$

The unexpected stereochemical course of the epoxidation of 4 thwarted our expectations to prepare diphosphines of general structure $\mathbf{I}$, since the preparation of compound $\mathbf{9}$ from epoxide $\mathbf{3}$ was not attempted taking into account the low stereoselectivity of the epoxidation reaction.

However, throughout the synthetic sequence, we prepared and fully characterized different cyclopentane phosphine oxides, whose relative configurations and conformations were deduced from the ${ }^{1} \mathrm{H}$ NMR data with the aid of DFT theoretical calculations. These compounds might be of interest as synthetic intermediates in phosphorus chemistry.

\section{Theoretical Calculations}

Initial optimizations were carried out using PCModel 8.0 Program. ${ }^{10}$ Further geometrical optimizations of model compounds 11, 12 and 13 were carried out at Becke's three-parameter hybrid functional with the Lee, Yang and Parr correlation functional (B3LYP) level ${ }^{11}$ using the standard $6-31 \mathrm{G}(\mathrm{d}, \mathrm{p})$ basis set. ${ }^{12}$ The minimum energy nature of the optimized structures was verified from vibrational frequency analysis (no negative vibrational frequencies). Coupling constants were calculated on the previously optimized geometries using the GIAO method ${ }^{13}$ with the NMR=SpinSpin option. ${ }^{14}$ All calculations were carried out with the Gaussian 03 on a Compaq HPC320 computer. ${ }^{15}$

\section{Experimental Section}

General Procedures. Melting points were determined with a MFB 595010 M Gallenkamp melting point apparatus. $500 \mathrm{MHz}{ }^{1} \mathrm{H}$ NMR spectra were recorded on a Varian VXR 500 spectrometer, 75.4 MHz ${ }^{13} \mathrm{C}$ NMR spectra were taken on a Varian Gemini 300 and $121.4 \mathrm{MHz}$ ${ }^{31} \mathrm{P}$ NMR on a Varian Unity 300 Plus, in $\mathrm{CDCl}_{3}$ solution, except where other solvent is indicated. ${ }^{1} \mathrm{H}$ and ${ }^{13} \mathrm{C}$ NMR chemical shifts $(\delta)$ are reported in ppm with respect to internal 
tetramethylsilane (TMS) and ${ }^{31} \mathrm{P}$ NMR chemical shifts $(\delta)$ are reported in ppm relative to $85 \%$ $\mathrm{H}_{3} \mathrm{PO}_{4}$ as external standard. The multiplicity of the signals is: $\mathrm{s}$, singulet; $d$, doublet; $\mathrm{t}$, triplet; $\mathrm{m}$, multiplet. For the different compounds, the terms $\mathrm{H}_{\alpha}$ or $\mathrm{H}_{\beta}$ are assigned to hydrogen atoms which are cis or trans relative to the diphenylphosphinoylmethyl group in 4, the epoxide group in the cases of $\mathbf{3}$ and $\mathbf{8}$, and the hydroxyl group in the case of 10. IR spectra were recorded on a FT/IR Perkin-Elmer spectrometer, model 1600; only significant absorption bands are given. Routine MS spectra were taken on a Hewlett-Packard 5988A spectrometer, the sample being introduced directly or through a gas chromatograph, Hewlett-Packard model 5890 Series II, equipped with a 30-meter HP-5 (5\% diphenyl-95\% dimethyl-polysiloxane) column and the electron impact technique $(70 \mathrm{eV})$. Only significant ions are given: those with higher relative abundance, except for the ions with higher $m / z$ values. NMR and routine MS spectra were performed at the Serveis Científico-Tècnics of the University of Barcelona, while elemental analyses were carried out at the Microanalysis Service of the IIQAB (CSIC, Barcelona, Spain).

3-[(Diphenylphosphinoyl)methyl]cyclopent-2-en-1-one (6). To a cold (ice-bath) solution of methyldiphenylphosphine oxide $(98 \%, 9.6 \mathrm{~g}, 44.4 \mathrm{mmol})$ in anhydrous THF (125 mL), $n$ butyllithium $(2.5 \mathrm{~mL}, 1.6 \mathrm{M}$ in hexanes, $4.0 \mathrm{mmol})$ was added dropwise till the solution became bright yellow. The solution was cooled to $-20^{\circ} \mathrm{C}$ and more $n$-butyllithium $(28 \mathrm{~mL}, 1.6 \mathrm{M}$ in hexanes, $45 \mathrm{mmol}$ ) was added. 3-Methoxycyclopent-2-en-1-one, 7 (5.00 g, $44.6 \mathrm{mmol}$ ), in anhydrous THF $(175 \mathrm{~mL})$ was added dropwise to the orange solution, and the mixture was allowed to warm to $-5{ }^{\circ} \mathrm{C}$. Water $(40 \mathrm{~mL}), 10 \%$ aqueous solution of $\mathrm{H}_{2} \mathrm{SO}_{4}(200 \mathrm{~mL})$ and EtOAc $(300 \mathrm{~mL})$ were added, and the mixture was stirred for $30 \mathrm{~min}$. The organic phase was separated and the aqueous one was evaporated to dryness in vacuo. The residue was taken in $\mathrm{H}_{2} \mathrm{O}(35 \mathrm{~mL})$ and the solution was extracted with EtOAc $(3 \times 200 \mathrm{~mL})$. The combined organic phases were washed with saturated aqueous solution of $\mathrm{NaHCO}_{3}(2 \times 75 \mathrm{~mL})$, dried (anhydrous $\mathrm{Na}_{2} \mathrm{SO}_{4}$ ), filtered and concentrated in vacuo to give $\mathbf{6}$ as an oil. Crystallization (EtOAc / hexanes) gave pure 6 (5.7 g, 43\% yield), whose ${ }^{1} \mathrm{H}$ and ${ }^{13} \mathrm{C}$ NMR spectra coincide with the data previously published. ${ }^{3}$

Tosylhydrazone of 3-[(Diphenylphosphinoyl)methyl]cyclopent-2-en-1-one (5). Solid 6 (5.50 $\mathrm{g}, 18.6 \mathrm{mmol}$ ) was dissolved in the minimum amount of acetic acid, tosylhydrazine (6.9 $\mathrm{g}, 37$ mmol) was added and the solution was stirred overnight at room temperature. The white precipitate was filtered and dried in vacuo to give pure 5 (7.00 g, 81\% yield) as a solid, m.p. $>220^{\circ} \mathrm{C}$ (dec.). Occasionally, the addition of water to induce the precipitation of 5 was required. IR (KBr) $3195(\mathrm{NH})$, 3055, 3025, $1611\left(\mathrm{C}=\mathrm{N}\right.$ st), $1333\left(\mathrm{SO}_{2}\right.$, st as), 1185 ( $\mathrm{P}=\mathrm{O}$ st), $1163\left(\mathrm{SO}_{2}\right.$, st si) $\mathrm{cm}^{-1} ;{ }^{1} \mathrm{H}$ NMR (DMSO-d $) 2.36\left(\mathrm{~s}, 3 \mathrm{H}, \mathrm{Ar}-\mathrm{CH}_{3}\right), 2.34-2.44$ (complex signal, 4H, 4-H, $5-$ $\mathrm{H}_{2}$ ), 3.64 (d, J=14.5 Hz, 2H, $\mathrm{CH}_{2}-\mathrm{P}$ ), 5.80 (broad s, 1H, 2-H), 7.35 [d, J=8.0 Hz, 2H, tolyl 3(5)H], 7.47-7.57 (complex signal, 6H, phenyl $\mathrm{H}_{\text {meta }}$ and $\mathrm{H}_{\text {para }}$ ), 7.67 [d, J=8.0 Hz, 2H, tolyl 2(6)H], 7.79-7.84 (complex signal, 4H, phenyl $\mathrm{H}_{\text {ortho}}$ ), 9.82 (broad s, $1 \mathrm{H}, \mathrm{NH}$ ); ${ }^{13} \mathrm{C} \mathrm{NMR}$ $\left(\mathrm{DMSO}_{6}\right) 21.2\left(\mathrm{CH}_{3}\right.$, tolyl $\left.\mathrm{CH}_{3}\right), 26.9\left(\mathrm{CH}_{2}, \mathrm{C} 5\right), 33.5\left(\mathrm{CH}_{2}, \mathrm{~d},{ }^{1} J_{\mathrm{C} / \mathrm{P}}=65.4 \mathrm{~Hz}, \mathrm{CH}_{2}-\mathrm{P}\right), 34.7$ $\left(\mathrm{CH}_{2}, \mathrm{~s}, \mathrm{C} 4\right), 127.6\left[\mathrm{CH}, \mathrm{s}\right.$, tolyl C3(5)], $128.9\left(\mathrm{CH}, \mathrm{d},{ }^{3} J_{\mathrm{C} / \mathrm{P}}=11.4 \mathrm{~Hz}\right.$, phenyl $\left.\mathrm{CH}_{\text {meta }}\right), 129.2(\mathrm{CH}$, $\left.\mathrm{d},{ }^{2} J_{\mathrm{C} / \mathrm{P}}=8.2 \mathrm{~Hz}, \mathrm{C} 2\right), 129.6\left[\mathrm{CH}, \mathrm{s}\right.$, tolyl C2(6)], $130.7\left(\mathrm{CH}, \mathrm{d},{ }^{2} J_{\mathrm{C} / \mathrm{P}}=9.1 \mathrm{~Hz}\right.$, phenyl $\left.\mathrm{CH}_{\text {ortho }}\right)$, 
$132.0\left(\mathrm{CH}, \mathrm{s}\right.$, phenyl $\left.\mathrm{CH}_{\text {para }}\right), 133.6\left(\mathrm{C}, \mathrm{d},{ }^{1} J_{\mathrm{C} / \mathrm{P}}=97.5 \mathrm{~Hz}\right.$, phenyl $\left.\mathrm{C}_{i p s o}\right), 136.5(\mathrm{C}, \mathrm{s}$, tolyl $\mathrm{C} 1)$, $143.3\left(\mathrm{C}, \mathrm{s}\right.$, tolyl C4), $155.2\left(\mathrm{C}, \mathrm{d},{ }^{2} J_{\mathrm{C} / \mathrm{P}}=9.9 \mathrm{~Hz}, \mathrm{C} 3\right), 167.9\left(\mathrm{C}, \mathrm{d},{ }^{4} J_{\mathrm{C} / \mathrm{P}}=3.4 \mathrm{~Hz}, \mathrm{C} 1\right)$; ${ }^{31} \mathrm{P}$ NMR (DMSO-d 6 ) 26.7; MS (EI), m/z (\%): 446 (11), 281 (37), 280 (57), 279 (15), 208 (12), 203 (14), 202 (100), $201\left[\left[\mathrm{PO}\left(\mathrm{C}_{6} \mathrm{H}_{5}\right)_{2}\right]^{+}, 82\right], 156$ (22), 155 (40), 92 (41), 91 (49), $77\left[\left(\mathrm{C}_{6} \mathrm{H}_{5}\right)^{+}, 42\right], 65$ (34), 51 (22). Anal. Calcd. for $\mathrm{C}_{25} \mathrm{H}_{25} \mathrm{~N}_{2} \mathrm{O}_{3} \mathrm{PS} \cdot 0.6 \mathrm{H}_{2} \mathrm{O}: \mathrm{C}, 63.17 ; \mathrm{H}, 5.42 ; \mathrm{N}, 5.89 ; \mathrm{S}, 6.75$. Found: C, 63.03; H, 5.30; N, 5.81; S, 6.56.

[(Cyclopent-2-en-1-yl)methyl]diphenylphosphine oxide (4). To a solution of 5 (7.00 g, 15.1 mmol) in acetic acid $(200 \mathrm{~mL}), \mathrm{NaBH}_{4}(5.7 \mathrm{~g}, 151 \mathrm{mmol})$ was added portionwise. The mixture was stirred at room temperature for $1 \mathrm{~h}$ and was heated under reflux for $3 \mathrm{~h}$. The mixture was added to ice and $5 \mathrm{~N} \mathrm{NaOH}$ was added till basic $\mathrm{pH}$. The aqueous phase was extracted with diethyl ether $(3 \times 300 \mathrm{~mL})$ and the combined organic extracts were dried (anhydrous $\mathrm{Na}_{2} \mathrm{SO}_{4}$ ), filtered and concentrated in vacuo. Column chromatography of the oily yellow residue (silica gel, EtOAc / hexanes mixtures) followed by crystallization (EtOAc) gave pure 4 (2.60 g, 60\% yield), as a solid, m.p. $124.5-126^{\circ} \mathrm{C}$. IR (KBr) 3050, 2924, 2858, 2846, 1437, 1180 (P=O st) $\mathrm{cm}^{-1} ;{ }^{1} \mathrm{H}$ NMR $1.48\left(\mathrm{ddt}, 1 \mathrm{H},{ }^{2} J_{5-\mathrm{H \alpha} / 5-\mathrm{H} \beta}=13.0 \mathrm{~Hz},{ }^{3} J_{5-\mathrm{H \alpha} / 4-\mathrm{H \alpha}}=9.0 \mathrm{~Hz},{ }^{3} J_{5-\mathrm{H \alpha} / 4-\mathrm{H} \beta}={ }^{3} J_{5-\mathrm{H \alpha} / 1-\mathrm{H}}=6.5 \mathrm{~Hz}\right.$, $\left.5-\mathrm{H}_{\alpha}\right), 2.05$ (ddt, $1 \mathrm{H},{ }^{2} J_{5-\mathrm{H} \beta / 5-\mathrm{H \alpha}}=13.0 \mathrm{~Hz},{ }^{3} J_{5-\mathrm{H} \beta / 4-\mathrm{H \alpha}}=8.5 \mathrm{~Hz},{ }^{3} J_{5-\mathrm{H} \beta / 4-\mathrm{H} \beta}={ }^{3} J_{5-\mathrm{H} \alpha / 1-\mathrm{H}}=4.5 \mathrm{~Hz}, 5-\mathrm{H}_{\beta}$ ), 2.16-2.33 (m, $\left.1 \mathrm{H}, 4-\mathrm{H}_{\beta}\right), 2.26-2.33$ (overlapped $\mathrm{m}\left(4-\mathrm{H}_{\alpha}\right)$ plus ddd, ${ }^{2} J_{\mathrm{CHa}-\mathrm{P} / \mathrm{CHb}-\mathrm{P}}=15.0 \mathrm{~Hz},{ }^{2} J_{\mathrm{CHa}}$ $\left.\mathrm{P} / \mathrm{P}=10.5 \mathrm{~Hz},{ }^{3} J_{\mathrm{CHa}-\mathrm{P} / 1-\mathrm{H}}=7.5 \mathrm{~Hz}, \mathrm{CH}_{\mathrm{a}}-\mathrm{P}\right), 2.40\left(\mathrm{ddd}, 1 \mathrm{H},{ }^{2} J_{\mathrm{CHa} / \mathrm{CHb}}=15.0 \mathrm{~Hz},{ }^{2} J_{\mathrm{CHb} / \mathrm{P}}=11 \mathrm{~Hz},{ }^{3} J_{\mathrm{CHb} / 1-}\right.$ $\left.\mathrm{H}_{\mathrm{H}}=6.5 \mathrm{~Hz}, \mathrm{CH}_{\mathrm{b}}-\mathrm{P}\right), 3.07(\mathrm{~m}, 1 \mathrm{H}, 1-\mathrm{H}), 5.61(\mathrm{~m}, 1 \mathrm{H}, 2-\mathrm{H}), 5.68(\mathrm{~m}, 1 \mathrm{H}, 3-\mathrm{H}), 7.42-7.51$ (complex signal, $6 \mathrm{H}$, phenyl $\mathrm{H}_{\text {meta }}$ and $\mathrm{H}_{\text {para }}$ ), $7.72-7.77$ (complex signal, $4 \mathrm{H}$, phenyl $\mathrm{H}_{\text {ortho}}$ ); ${ }^{13} \mathrm{C}$ NMR $31.6\left(\mathrm{CH}_{2}, \mathrm{~d},{ }^{3} J_{\mathrm{C} / \mathrm{P}}=8.6 \mathrm{~Hz}, \mathrm{C} 5\right), 31.7\left(\mathrm{CH}_{2}, \mathrm{~s}, \mathrm{C} 4\right), 35.9\left(\mathrm{CH}_{2}, \mathrm{~d},{ }^{1} J_{\mathrm{C} / \mathrm{P}}=70.6 \mathrm{~Hz}, \mathrm{CH}_{2}-\mathrm{P}\right), 39.1$ $\left(\mathrm{CH}, \mathrm{d},{ }^{2} J_{\mathrm{C} / \mathrm{P}}=3.6 \mathrm{~Hz}, \mathrm{C} 1\right), 128.5\left(\mathrm{CH}, \mathrm{d},{ }^{3} J_{\mathrm{C} / \mathrm{P}}=11.5 \mathrm{~Hz}\right.$, phenyl $\left.\mathrm{CH}_{\text {meta }}\right), 130.7\left(\mathrm{CH}, \mathrm{d},{ }^{2} J_{\mathrm{C} / \mathrm{P}}=9.3\right.$ $\mathrm{Hz}$, phenyl $\left.\mathrm{CH}_{\text {ortho }}\right), 131.1(\mathrm{CH}, \mathrm{s}, \mathrm{C} 3), 131.5\left(\mathrm{CH}\right.$, phenyl $\left.\mathrm{CH}_{\text {para }}\right), 133.5\left(\mathrm{C}, \mathrm{d},{ }^{1} J_{\mathrm{C} / \mathrm{P}}=97.3 \mathrm{~Hz}\right)$ and $133.6\left(\mathrm{C}, \mathrm{d},{ }^{1} J_{\mathrm{C} / \mathrm{P}}=97.4 \mathrm{~Hz}\right)$ (phenyl $\left.\mathrm{C}_{i p s o}\right), 134.5\left(\mathrm{CH}, \mathrm{d},{ }^{3} J_{\mathrm{C} / \mathrm{P}}=9.4 \mathrm{~Hz}, \mathrm{C} 2\right) ;{ }^{31} \mathrm{P}$ NMR 30.6; MS (EI), m/z (\%): $284(24), 283\left[(\mathrm{M}+\mathrm{H})^{+}, 100\right], 216(23), 215\left[\left[\mathrm{CH}_{2} \mathrm{PO}\left(\mathrm{C}_{6} \mathrm{H}_{5}\right)_{2}\right]^{+}, 16\right], 205[(\mathrm{M}-$ $\left.\left.\mathrm{C}_{6} \mathrm{H}_{5}\right)^{+}, 23\right], 202\left[\left(\mathrm{HPO}\left(\mathrm{C}_{6} \mathrm{H}_{5}\right)_{2}{ }^{+}, 18\right]\right.$. Anal. Calcd. for $\mathrm{C}_{18} \mathrm{H}_{19} \mathrm{OP}: \mathrm{C}, 76.58 ; \mathrm{H}, 6.78$. Found: C, $76.50 ; \mathrm{H}, 6.70$.

Syn- and anti-[(2,3-epoxycyclopent-1-yl)methyl]diphenylphosphine oxide (8 and 3). To a solution of $4(2.50 \mathrm{~g}, 8.87 \mathrm{mmol})$ in $\mathrm{CH}_{2} \mathrm{Cl}_{2}(75 \mathrm{~mL})$ was added $\mathrm{m}$-CPBA $(7.6 \mathrm{~g}, 40 \%$ content, $17.6 \mathrm{mmol}$ ) and the suspension was stirred at room temperature for $18 \mathrm{~h}$. The suspension was basified with aqueous $5 \mathrm{~N} \mathrm{NaOH}(75 \mathrm{~mL})$, the organic phase was separated and the aqueous one was extracted with $\mathrm{CH}_{2} \mathrm{Cl}_{2}(3 \times 75 \mathrm{~mL})$. The combined organic phases were dried (anhydrous $\left.\mathrm{Na}_{2} \mathrm{SO}_{4}\right)$, filtered and concentrated in vacuo to give a white solid $(2.00 \mathrm{~g})$. Column chromatography of this solid (silica gel, EtOAc / methanol mixtures) gave pure 8 (1.6 g, 61\% yield) and 3 (0.24 g, 9\% yield). An analytical sample of 8 was obtained by crystallization (EtOAc), as a solid, m.p. $146-147^{\circ} \mathrm{C}$. IR (KBr) 3054, 2945, 1438, $1178\left(\mathrm{P}=\mathrm{O}\right.$ st) cm ${ }^{-1}$; ${ }^{1} \mathrm{H}$ NMR $0.96\left(\mathrm{ddt},{ }^{2} J_{5-\mathrm{H} \alpha / 5-\mathrm{H} \beta}=12.5 \mathrm{~Hz},{ }^{3} J_{5-\mathrm{H} \alpha / 4-\mathrm{H \alpha}}=8.0 \mathrm{~Hz},{ }^{3} J_{5-\mathrm{H} \alpha / 4-\mathrm{H} \beta}={ }^{3} J_{5-\mathrm{H} \alpha / 1-\mathrm{H}}=10.0 \mathrm{~Hz}, 1 \mathrm{H}, 5-\mathrm{H}_{\alpha}\right), 1.51$ (dddd, ${ }^{2} J_{4-\mathrm{H} \beta / 4-\mathrm{H \alpha}}=14.0 \mathrm{~Hz},{ }^{3} J_{4-\mathrm{H} \beta / 5-\mathrm{H \alpha}}=10.0 \mathrm{~Hz},{ }^{3} J_{4-\mathrm{H} \beta / 5-\mathrm{H} \beta}=8.5 \mathrm{~Hz},{ }^{3} J_{4-\mathrm{H} \beta / 3-\mathrm{H}}=2.0 \mathrm{~Hz}, 1 \mathrm{H}, 4-\mathrm{H}_{\beta}$ ), $1.59\left(\mathrm{dt},{ }^{2} J_{5-\mathrm{H} \beta / 5-\mathrm{H \alpha}}=12.5 \mathrm{~Hz},{ }^{3} J_{5-\mathrm{H} \beta / 4-\mathrm{H} \beta}={ }^{3} J_{5-\mathrm{H} \beta / 1-\mathrm{H}}=8.5 \mathrm{~Hz}, 1 \mathrm{H}, 5-\mathrm{H}_{\beta}\right), 1.93\left(\mathrm{dd},{ }^{2} J_{4-\mathrm{H} \alpha / 4-\mathrm{H} \beta}=14.0 \mathrm{~Hz}\right.$, $\left.{ }^{3} J_{4-\mathrm{H} \alpha / 5-\mathrm{H} \alpha}=8.0 \mathrm{~Hz}, 1 \mathrm{H}, 4-\mathrm{H}_{\alpha}\right), 2.38\left(\mathrm{ddd},{ }^{2} J_{\mathrm{CHa}-\mathrm{P} / \mathrm{CHb}-\mathrm{P}}=14.5 \mathrm{~Hz},{ }^{2} J_{\mathrm{CHa}-\mathrm{P} / \mathrm{P}}=10.5 \mathrm{~Hz},{ }^{3} J_{\mathrm{CHa}-\mathrm{P} / 1-\mathrm{H}}=6.5\right.$ 
$\mathrm{Hz}, 1 \mathrm{H}, \mathrm{CH}_{\mathrm{a}}-\mathrm{P}$ ), 2.61 (ddd, ${ }^{2} J_{\mathrm{CHb}-\mathrm{P} / \mathrm{CHa}-\mathrm{P}}=14.5 \mathrm{~Hz},{ }^{2} J_{\mathrm{CHb}-\mathrm{P} / \mathrm{P}}=10.0 \mathrm{~Hz},{ }^{3} J_{\mathrm{CHb}-\mathrm{P} / 1-\mathrm{H}}=7.0 \mathrm{~Hz}, 1 \mathrm{H}, \mathrm{CH}_{\mathrm{b}^{-}}$ P), $2.46(\mathrm{~m}, 1 \mathrm{H}, 1-\mathrm{H}), 3.21\left(\operatorname{broad~d},{ }^{3} J_{2-\mathrm{H} / 1-\mathrm{H}}=2.5 \mathrm{~Hz}, 1 \mathrm{H}, 2-\mathrm{H}\right), 3.34\left(\operatorname{broad~d},{ }^{3} J_{3-\mathrm{H} / 4-\mathrm{H} \beta}=2.0 \mathrm{~Hz}\right.$, 1H, 3-H), 7.41-7.51 (complex signal, 6H, phenyl $\mathrm{H}_{\text {meta }}$ and $\mathrm{H}_{\text {para }}$ ), 7.71-7.80 (complex signal, $4 \mathrm{H}$, phenyl $\left.\mathrm{H}_{\text {ortho }}\right) ;{ }^{13} \mathrm{C}$ NMR $26.4\left(\mathrm{CH}_{2}, \mathrm{~d},{ }^{3} J_{\mathrm{C} / \mathrm{P}}=8.6 \mathrm{~Hz}, \mathrm{C} 5\right), 27.1\left(\mathrm{CH}_{2}, \mathrm{~s}, \mathrm{C} 4\right), 31.2\left(\mathrm{CH}_{2}, \mathrm{~d}\right.$, $\left.{ }^{1} J_{\mathrm{C} / \mathrm{P}}=71.5 \mathrm{~Hz}, \mathrm{CH}_{2}-\mathrm{P}\right), 33.9\left(\mathrm{CH}, \mathrm{d},{ }^{2} J_{\mathrm{C} / \mathrm{P}}=3.7 \mathrm{~Hz}, \mathrm{C} 1\right), 57.4(\mathrm{CH}, \mathrm{s}, \mathrm{C} 3), 59.6\left(\mathrm{CH}, \mathrm{d},{ }^{3} J_{\mathrm{C} / \mathrm{P}}=8.3\right.$ $\mathrm{Hz}, \mathrm{C} 2), 128.5\left(\mathrm{CH}, \mathrm{d},{ }^{3} J_{\mathrm{C} / \mathrm{P}}=11.6 \mathrm{~Hz}\right)$ and $128.6\left(\mathrm{CH}, \mathrm{d},{ }^{3} J_{\mathrm{C} / \mathrm{P}}=11.6 \mathrm{~Hz}\right)\left(\right.$ phenyl $\left.\mathrm{CH}_{\text {meta }}\right), 130.5$ $\left(\mathrm{CH}, \mathrm{d},{ }^{2} J_{\mathrm{C} / \mathrm{P}}=9.1 \mathrm{~Hz}\right.$, phenyl $\left.\mathrm{CH}_{\text {ortho }}\right), 131.6\left(\mathrm{CH}, \mathrm{d},{ }^{4} J_{\mathrm{C} / \mathrm{P}}=2.3 \mathrm{~Hz}\right)$ and $131.6\left(\mathrm{CH}, \mathrm{d},{ }^{4} J_{\mathrm{C} / \mathrm{P}}=2.3\right.$ $\mathrm{Hz}$ ) (phenyl $\mathrm{CH}_{\text {para }}$ ), $133.2\left(\mathrm{C}, \mathrm{d},{ }^{1} J_{\mathrm{C} / \mathrm{P}}=97.9 \mathrm{~Hz}\right)$ and $133.3\left(\mathrm{C}, \mathrm{d},{ }^{1} J_{\mathrm{C} / \mathrm{P}}=97.9 \mathrm{~Hz}\right)$ (phenyl $\mathrm{C}_{\text {ipso }}$ ); ${ }^{31} \mathrm{P}$ NMR 32.9; MS (EI), m/z (\%): $298\left(\mathrm{M}^{+}, 2\right), 216(42), 215\left\{\left[\mathrm{CH}_{2} \mathrm{PO}\left(\mathrm{C}_{6} \mathrm{H}_{5}\right)_{2}\right]^{+}, 100\right\}, 203$ (12), 202 (59), $201\left\{\left[\mathrm{PO}\left(\mathrm{C}_{6} \mathrm{H}_{5}\right)_{2}\right]^{+}, 57\right\}, 156(50), 77\left[\left(\mathrm{C}_{6} \mathrm{H}_{5}\right)^{+}, 35\right]$. Anal. Calcd. for $\mathrm{C}_{18} \mathrm{H}_{19} \mathrm{O}_{2} \mathrm{P}$ : C, 72.47; H, 6.42. Found: C, 72.50; H, 6.41. An analytical sample of 3 was obtained by crystallization (EtOAc), as a solid, m.p. $144-145^{\circ} \mathrm{C}$. IR $(\mathrm{KBr}) 3054,1438,1180(\mathrm{P}=\mathrm{O} \mathrm{st}) \mathrm{cm}^{-1}$; ${ }^{1} \mathrm{H}$ NMR $1.49\left(\mathrm{dd},{ }^{2} J_{5-\mathrm{H} \alpha / 5-\mathrm{H} \beta}=13.0 \mathrm{~Hz},{ }^{3} J_{5-\mathrm{H} \beta / 4-\mathrm{H} \beta}=9.0 \mathrm{~Hz}, 1 \mathrm{H}, 5-\mathrm{H}_{\beta}\right), 1.56\left(\mathrm{~m}, 1 \mathrm{H}, 5-\mathrm{H}_{\alpha}\right), 1.66$ (dddd, ${ }^{2} J_{4-\mathrm{H \alpha} / 4-\mathrm{H} \beta}=14.0 \mathrm{~Hz},{ }^{3} J_{4-\mathrm{H} \beta / 5-\mathrm{H \alpha}}=10.0 \mathrm{~Hz},{ }^{3} J_{4-\mathrm{H} \beta / 5-\mathrm{H} \beta}=9.0 \mathrm{~Hz},{ }^{3} J_{4-\mathrm{H} \beta / 3-\mathrm{H}}=1.0 \mathrm{~Hz}, 1 \mathrm{H}, 4-\mathrm{H}_{\beta}$ ), $1.96\left(\mathrm{dd},{ }^{2} J_{4-\mathrm{H} \alpha / 4-\mathrm{H} \beta}=14.0 \mathrm{~Hz},{ }^{3} J_{4-\mathrm{H} \alpha / 5-\mathrm{H} \alpha}=7.5 \mathrm{~Hz}, 1 \mathrm{H}, 4-\mathrm{H}_{\alpha}\right), 2.14$ (ddd, ${ }^{2} J_{\mathrm{CHa}-\mathrm{P} / \mathrm{CHb}-\mathrm{P}}=15.0 \mathrm{~Hz}$, $\left.{ }^{2} J_{\mathrm{CHa}-\mathrm{P} / \mathrm{P}}=10.5 \mathrm{~Hz},{ }^{3} J_{\mathrm{CHa}-\mathrm{P} / 1-\mathrm{H}}=7.0 \mathrm{~Hz}, 1 \mathrm{H}, \mathrm{CH}_{\mathrm{a}}-\mathrm{P}\right), 2.23\left(\mathrm{ddd},{ }^{2} J_{\mathrm{CHb}-\mathrm{P} / \mathrm{CHa}-\mathrm{P}}=15.0 \mathrm{~Hz},{ }^{2} J_{\mathrm{CHb}-\mathrm{P} / \mathrm{P}}=12.0\right.$ $\left.\mathrm{Hz},{ }^{3} J_{\mathrm{CHb}-\mathrm{P} / 1-\mathrm{H}}=7.0 \mathrm{~Hz}, 1 \mathrm{H}, \mathrm{CH}_{\mathrm{b}}-\mathrm{P}\right), 2.58\left(\mathrm{dq},{ }^{3} J_{1-\mathrm{H} / \mathrm{P}}=11.0 \mathrm{~Hz},{ }^{3} J_{1-\mathrm{H} / \mathrm{CHa}-\mathrm{P}}={ }^{3} J_{1-\mathrm{H} / \mathrm{CHb}-\mathrm{P}}={ }^{3} J_{1-\mathrm{H} / 5 \mathrm{H \alpha}}=7.0\right.$ $\mathrm{Hz}, 1 \mathrm{H}, 1-\mathrm{H}), 3.46\left(\mathrm{~d}, 1 \mathrm{H},{ }^{3} J_{3-\mathrm{H} / 2-\mathrm{H}}=2.0,1 \mathrm{H}, 3-\mathrm{H}\right), 3.53\left(\mathrm{~d},{ }^{3} J_{2-\mathrm{H} / 3-\mathrm{H}}=2.0,1 \mathrm{H}, 2-\mathrm{H}\right), 7.46-7.56$ (complex signal, $6 \mathrm{H}$, phenyl $\mathrm{H}_{\text {meta }}$ and $\mathrm{Ar}-\mathrm{H}_{\text {para }}$ ), 7.74-7.80 (complex signal, $4 \mathrm{H}$, phenyl $\mathrm{H}_{\text {ortho); }}$ ) ${ }^{13} \mathrm{C}$ NMR $25.1\left(\mathrm{CH}_{2}, \mathrm{~s}, \mathrm{C} 4\right), 25.9\left(\mathrm{CH}_{2}, \mathrm{~d},{ }^{3} J_{\mathrm{C} / \mathrm{P}}=8.6 \mathrm{~Hz}, \mathrm{C} 5\right), 31.3\left(\mathrm{CH}_{2}, \mathrm{~d},{ }^{1} J_{\mathrm{C} / \mathrm{P}}=71.3 \mathrm{~Hz}, \mathrm{CH}_{2^{-}}\right.$ $\mathrm{P}), 33.1\left(\mathrm{CH}, \mathrm{d},{ }^{2} J_{\mathrm{C} / \mathrm{P}}=3.3 \mathrm{~Hz}, \mathrm{C} 1\right), 56.7(\mathrm{CH}, \mathrm{s}, \mathrm{C} 3), 60.1\left(\mathrm{CH}, \mathrm{d},{ }^{3} J_{\mathrm{C} / \mathrm{P}}=11.5 \mathrm{~Hz}, \mathrm{C} 2\right), 128.7(\mathrm{CH}$, $\left.\mathrm{d},{ }^{3} J_{\mathrm{C} / \mathrm{P}}=11.6 \mathrm{~Hz}, \mathrm{Ar}-\mathrm{CH}_{\text {meta }}\right), 130.6\left(\mathrm{CH}, \mathrm{d},{ }^{2} J_{\mathrm{C} / \mathrm{P}}=9.1 \mathrm{~Hz}\right)$ and $130.7\left(\mathrm{CH}, \mathrm{d},{ }^{2} J_{\mathrm{C} / \mathrm{P}}=9.1 \mathrm{~Hz}\right)(\mathrm{Ar}-$ $\left.\mathrm{CH}_{\text {ortho }}\right), 131.8\left(\mathrm{CH}, \mathrm{d},{ }^{4} J_{\mathrm{C} / \mathrm{P}}=2.8 \mathrm{~Hz}, \mathrm{Ar}-\mathrm{CH}_{\text {para }}\right), 132.8\left(\mathrm{C}, \mathrm{d},{ }^{1} J_{\mathrm{C} / \mathrm{P}}=98.6 \mathrm{~Hz}\right)$ and $133.0(\mathrm{C}, \mathrm{d}$, $\left.{ }^{1} J_{\mathrm{C} / \mathrm{P}}=98.6 \mathrm{~Hz}\right)\left(\mathrm{Ar}_{-} \mathrm{C}_{i p s o}\right) ;{ }^{31} \mathrm{P}$ NMR 30.9; MS (EI), m/z (\%): $298\left(\mathrm{M}^{+}, 6\right), 297$ (11), $216(51), 215$ $\left\{\left[\mathrm{CH}_{2} \mathrm{PO}\left(\mathrm{C}_{6} \mathrm{H}_{5}\right)_{2}\right]^{+}, 100\right\}, 202(63), 201\left\{\left[\mathrm{PO}\left(\mathrm{C}_{6} \mathrm{H}_{5}\right)_{2}\right]^{+}, 52\right\}, 155(25), 77\left[\left(\mathrm{C}_{6} \mathrm{H}_{5}\right)^{+}, 47\right], 51(31)$. Anal. Calcd. for $\mathrm{C}_{18} \mathrm{H}_{19} \mathrm{O}_{2} \mathrm{P}: \mathrm{C}, 72.47 ; \mathrm{H}, 6.42$. Found: $\mathrm{C}, 72.36 ; \mathrm{H}, 6.40$.

\section{(1r,2t,5t)-2-Diphenylphosphinoyl-5-[(diphenylphosphinoyl)methyl]cyclopentanol}

(10).

Chlorodiphenylphosphine $(1.5 \mathrm{~mL}, 8.1 \mathrm{mmol})$ was added to a boiling suspension of molten sodium $(0.40 \mathrm{~g}, 17 \mathrm{mmol})$ in anhydrous 1,4-dioxane $(25 \mathrm{~mL})$ and the suspension was heated under reflux for $3 \mathrm{~h}$. The yellow suspension was cooled to $0{ }^{\circ} \mathrm{C}$, was diluted with anhydrous THF $(25 \mathrm{~mL})$ and was further cooled to $-30^{\circ} \mathrm{C}$. A solution of $8(0.60 \mathrm{~g}, 2.0 \mathrm{mmol})$ in anhydrous THF $(5 \mathrm{~mL})$ was added and the mixture was stirred at $-30{ }^{\circ} \mathrm{C}$ overnight. The mixture was filtered through Celite ${ }^{\circledR}$ and the filtrate was concentrated in vacuo to give an oily residue. This residue was taken in methanol $(20 \mathrm{~mL})$, was cooled with an ice-bath and a $30 \%$ solution of $\mathrm{H}_{2} \mathrm{O}_{2}(5 \mathrm{~mL})$ was added. The suspension was stirred for $1 \mathrm{~h}$ at $0{ }^{\circ} \mathrm{C}$, for another $1 \mathrm{~h}$ at room temperature and then it was concentrated to dryness under reduced pressure. The residue was taken in EtOAc (50 $\mathrm{mL}$ ) and was washed with brine, was dried (anhydrous $\mathrm{Na}_{2} \mathrm{SO}_{4}$ ), filtered and the filtrate was concentrated in vacuo. Column chromatography of the waxy residue (silica gel, EtOAc / methanol mixtures) gave pure 10 (0.80 g, 80\% yield). An analytical sample of 10 was obtained by crystallization (EtOAc), as a solid, m.p. 86-88 ${ }^{\circ} \mathrm{C}$. IR (KBr) 3279 (O-H st), 3054, 2928, 1437 , 
$1174(\mathrm{P}=\mathrm{O}$ st $) \mathrm{cm}^{-1} ;{ }^{1} \mathrm{H}$ NMR $1.64\left(\mathrm{~m}, 1 \mathrm{H}, 4-\mathrm{H}_{\alpha}\right), 1.73\left(\mathrm{~m}, 1 \mathrm{H}, 3-\mathrm{H}_{\alpha}\right), 1.77-1.86$ (complex signal, $\left.2 \mathrm{H}, 3-\mathrm{H}_{\beta}, 4-\mathrm{H}_{\beta}\right), 1.98(\mathrm{~m}, 1 \mathrm{H}, 5-\mathrm{H}), 2.42\left(\mathrm{ddd}, 1 \mathrm{H},{ }^{2} J_{\mathrm{CHa}-\mathrm{P} / \mathrm{CHb}-\mathrm{P}}=15.0 \mathrm{~Hz},{ }^{2} J_{\mathrm{CHa}-\mathrm{P} / \mathrm{P}}=7.0 \mathrm{~Hz}\right.$, $\left.{ }^{3} J_{\mathrm{CHa}-\mathrm{P} / 5-\mathrm{H}}=5.0 \mathrm{~Hz}, \mathrm{CH}_{\mathrm{a}}-\mathrm{P}\right), 2.50\left(\mathrm{dt}, 1 \mathrm{H},{ }^{2} J_{\mathrm{CHa}-\mathrm{P} / \mathrm{CHb}-\mathrm{P}}=15.0 \mathrm{~Hz},{ }^{2} J_{\mathrm{CHb}-\mathrm{P} / \mathrm{P}}={ }^{3} J_{\mathrm{CHb}-\mathrm{P} / 5-\mathrm{H}}=11.0 \mathrm{~Hz}\right.$, $\left.\mathrm{CH}_{\mathrm{b}}-\mathrm{P}\right), 2.90$ (ddt, $\left.1 \mathrm{H},{ }^{2} J_{2-\mathrm{H} / 2-\mathrm{P}}=6.0 \mathrm{~Hz},{ }^{3} J_{2-\mathrm{H} / 1-\mathrm{H}}=3.0 \mathrm{~Hz},{ }^{3} J_{2-\mathrm{H} / 3-\mathrm{H \alpha}}={ }^{3} J_{2-\mathrm{H} / 3-\mathrm{H} \beta}=9.0 \mathrm{~Hz}, 2-\mathrm{H}\right), 4.58$ (ddd, $1 \mathrm{H},{ }^{3} J_{1-\mathrm{H} / 2-\mathrm{P}}=13.5 \mathrm{~Hz},{ }^{3} J_{1-\mathrm{H} / 5-\mathrm{H} \beta}=6.0 \mathrm{~Hz},{ }^{3} J_{1-\mathrm{H} / 2-\mathrm{H}}=3.0 \mathrm{~Hz}, 1-\mathrm{H}$ ), 4.9 (broad s, $1 \mathrm{H}, \mathrm{OH}$ ), 7.3-7.8 (complex signal, 20H, Ar-H); ${ }^{13} \mathrm{C}$ NMR $24.4\left(\mathrm{CH}_{2}, \mathrm{~s}, \mathrm{C} 3\right), 29.6\left(\mathrm{CH}_{2}, \mathrm{~d},{ }^{1} J_{\mathrm{C} / \mathrm{P}}=63.6 \mathrm{~Hz}\right.$, $\left.\mathrm{CH}_{2}-\mathrm{P}\right), 33.1\left(\mathrm{CH}_{2}, \mathrm{dd},{ }^{3} J_{\mathrm{C} / \mathrm{P}}=14.0 \mathrm{~Hz},{ }^{3} J_{\mathrm{C} / \mathrm{P}}=8.1 \mathrm{~Hz}, \mathrm{C} 4\right), 41.2\left(\mathrm{CH}\right.$, pseudo $\mathrm{t},{ }^{2} J_{\mathrm{C} / \mathrm{P}}={ }^{3} J_{\mathrm{C} / \mathrm{P}}=5.0 \mathrm{~Hz}$, C5), $45.7\left(\mathrm{CH}, \mathrm{d},{ }^{1} J_{\mathrm{C} / \mathrm{P}}=73.1 \mathrm{~Hz}, \mathrm{C} 2\right), 72.3\left(\mathrm{CH}\right.$, pseudo $\left.\mathrm{t},{ }^{2} J_{\mathrm{C} / \mathrm{P}}={ }^{3} J_{\mathrm{C} / \mathrm{P}}=3.0 \mathrm{~Hz}, \mathrm{C} 1\right), 128.3(\mathrm{CH}, \mathrm{d}$, ${ }^{3} J_{\mathrm{C} / \mathrm{P}}=11.4 \mathrm{~Hz}$ ) and $128.4\left(\mathrm{CH}, \mathrm{d},{ }^{3} J_{\mathrm{C} / \mathrm{P}}=11.6 \mathrm{~Hz}\right)$ (diphenylphosphorylmethyl $\left.\mathrm{CH}_{\text {meta }}\right), 128.6(\mathrm{CH}$, $\mathrm{d},{ }^{3} J_{\mathrm{C} / \mathrm{P}}=16.1 \mathrm{~Hz}$ ) and $128.7\left(\mathrm{CH}, \mathrm{d},{ }^{3} J_{\mathrm{C} / \mathrm{P}}=16.1 \mathrm{~Hz}\right.$ ) (diphenylphosphoryl $\left.\mathrm{CH}_{\text {meta }}\right), 130.2(\mathrm{CH}, \mathrm{d}$, $\left.{ }^{2} J_{\mathrm{C} / \mathrm{P}}=9.7 \mathrm{~Hz}\right), 130.6\left(\mathrm{CH}, \mathrm{d},{ }^{2} J_{\mathrm{C} / \mathrm{P}}=9.3 \mathrm{~Hz}\right), 130.7\left(\mathrm{CH}, \mathrm{d},{ }^{2} J_{\mathrm{C} / \mathrm{P}}=9.2 \mathrm{~Hz}\right)$ and $131.1(\mathrm{CH}, \mathrm{d}$, $\left.{ }^{2} J_{\mathrm{C} / \mathrm{P}}=9.1 \mathrm{~Hz}\right)\left(\mathrm{Ar}-\mathrm{CH}_{\text {ortho }}\right), 131.3\left(\mathrm{CH}, \mathrm{d},{ }^{4} J_{\mathrm{C} / \mathrm{P}}=3.0 \mathrm{~Hz}\right), 131.4\left(\mathrm{CH}, \mathrm{d},{ }^{4} J_{\mathrm{C} / \mathrm{P}}=3.0 \mathrm{~Hz}\right), 131.8(\mathrm{CH}$, $\left.\mathrm{d},{ }^{4} J_{\mathrm{C} / \mathrm{P}}=3.0 \mathrm{~Hz}\right)$ and $132.0\left(\mathrm{CH}, \mathrm{d},{ }^{4} J_{\mathrm{C} / \mathrm{P}}=3.0 \mathrm{~Hz}\right)\left(\mathrm{Ar}-\mathrm{CH}_{\text {para }}\right), 130.6\left(\mathrm{C}, \mathrm{d},{ }^{1} J_{\mathrm{C} / \mathrm{P}}=98.5 \mathrm{~Hz}\right), 132.2$ $\left(\mathrm{C}, \mathrm{d},{ }^{1} J_{\mathrm{C} / \mathrm{P}}=98.2 \mathrm{~Hz}\right), 132.9\left(\mathrm{C}, \mathrm{d},{ }^{1} J_{\mathrm{C} / \mathrm{P}}=97.3 \mathrm{~Hz}\right)$, and $133.1\left(\mathrm{C}, \mathrm{d},{ }^{1} J_{\mathrm{C} / \mathrm{P}}=99.1 \mathrm{~Hz}\right)\left(\mathrm{Ar}_{-} \mathrm{C}_{i p s o}\right) ;{ }^{31} \mathrm{P}$ NMR 36.6, 37.2; MS (EI), m/z (\%): 500 (M+1, 1), 472 (15), 300 (20), 299 (98), 271 (46), 229 (12), $215\left\{\left[\mathrm{CH}_{2} \mathrm{PO}\left(\mathrm{C}_{6} \mathrm{H}_{5}\right)_{2}\right]^{+}, 12\right\}, 202(32), 201\left[\left[\mathrm{PO}\left(\mathrm{C}_{6} \mathrm{H}_{5}\right)_{2}\right]^{+}, 100\right], 77\left[\left(\mathrm{C}_{6} \mathrm{H}_{5}\right)^{+}, 23\right]$. Anal. Calcd. for $\mathrm{C}_{30} \mathrm{H}_{30} \mathrm{O}_{3} \mathrm{P}_{2}$ : C, 71.99; H, 6.04. Found: $\mathrm{C}, 71.93 ; \mathrm{H}, 6.02$.

\section{Acknowledgements}

Financial support from Ministerio de Ciencia y Tecnología (Project PPQ2002-01080) and Comissionat per a Universitats i Recerca (Project 2005-SGR-00180) is gratefully acknowledged. G. C. thanks to the Generalitat de Catalunya (Beca predoctoral). We thank the Centre de Supercomputació de Catalunya (CESCA) for computational facilities, the Serveis CientíficoTècnics of the University of Barcelona for performing the NMR spectra and Ms. P. Domènech from the IIQAB (Barcelona, Spain) for carrying out the elemental analyses.

\section{References and Footnotes}

1. (a) Morimoto, T.; Takahashi, H.; Achiwa, K. Chem. Pharm. Bull. 1994, 42, 481. (b) Sakuraba, S.; Takahashi, H.; Takeda, H.; Achiwa, K. Chem. Pharm. Bull. 1995, 43, 738. (c) Takeda, H.; Tachinami, T.; Aburatani, M.; Takahashi, H.; Morimoto, T.; Achiwa, K. Tetrahedron Lett. 1989, 30, 363. (d) Takahashi, H.; Sakuraba, S.; Takeda, H.; Achiwa, K. J. Am. Chem. Soc. 1990, 112, 5876. (e) Sakuraba, S.; Nakajima, N.; Achiwa, K. Tetrahedron: Asymmetry 1993, 4, 1457. (f) Sakuraba, S.; Achiwa, K. Synlett 1991, 689.

2. (a) Camps, P.; Colet, G.; Font-Bardia, M.; Muñoz-Torrero, V.; Solans, X.; Vázquez, S. Tetrahedron 2002, 58, 3473. (b) Camps, P.; Colet, G.; Font-Bardia, M.; Muñoz-Torrero, V.; 
Solans, X.; Vázquez, S. Tetrahedron: Asymmetry 2002, 13, 759. (c) Camps, P.; Colet, G.; Vázquez S. Arkivoc 2003, X, 16.

3. Öhler, E.; Zbiral, E. Synthesis 1991, 357.

4. (a) Caglioti, L.; Magi, M. Tetrahedron 1963, 19, 1127. (b) Hutchins R. O.; Milewski, B. E.; Maryanoff, B. E. J. Am. Chem. Soc. 1973, 95, 3662. (c) Hutchins R. O.; Kacher, M.; Rua, L. J. Org. Chem. 1975, 40, 923. (d) Taylor, E. J.; Djerassi, C. J. Am. Chem. Soc. 1976, 98, 2275. (e) Kabalka, G. W.; Baker, Jr., J. D. J. Org. Chem. 1975, 40, 1834. (f) Kabalka, G. W.; Yang, D. T. C.; Chandler, J. H.; Baker, Jr., J. D. Synthesis 1977, 124. (g) Kabalka, G. W.; Yang, D. T. C.; Baker, Jr., J. D. J. Org. Chem. 1976, 41, 574. (h) Natale, N. R.; Hutchins, R. O. Org. Prep. Proced. Int. 1979, 11, 201. (i) Kabalka, G. W. Org. Prep. Proced. Int. 1977, 9, 131. For a very recent application in total synthesis, see: Hong, S.; Corey, E. J. J. Am. Chem. Soc. 2006, 128, 1346.

5. Hutchins, R. O.; Natale, N. R. J. Org. Chem. 1978, 43, 2299.

6. The epoxidation of allylic phosphine oxides has been extensively studied by Warren and coworkers, but few work has been done on the epoxidation of homoallylic phosphine oxides. See (a) Clayden, J.; Collington, E. W.; Egert, E.; McElroy, A. B.; Warren, S. J. Chem. Soc., Perkin Trans. 1 1994, 2801. (b) Clayden, J.; Warren, S. J. Chem. Soc., Perkin Trans. 1 1994, 2811. (c) Nelson, A.; Warren, S. J. Chem. Soc., Perkin Trans. 1 1999, 1983. (d) Clayden, J.; Warren, S. Angew. Chem. Int. Ed. Engl. 1996, 35, 241.

7. Adam, W.; Bialas, J.; Hadjiarapoglou, L. Chem. Ber. 1991, 124, 2377.

8. (a) Issleib, K.; Möbius, H. M. Chem. Ber. 1961, 94, 102. (b) Oehme, H.; Issleib, K.; Leissring, E. Tetrahedron 1972, 28, 2587.

9. (a) Li, C.; Bernet, B.; Vasella, A.; Broger, E. A.; Meili, A. Carbohydr. Res. 1991, $216,149$. (b) Brunner, H.; Sicheneder, A. Angew. Chem., Int. Ed. Engl. 1988, 27, 718. (c) Boeckh, D.; Huisgen, R.; Nöth, H. J. Am. Chem. Soc. 1987, 109, 1248.

10. Serena Software, PCModel, Version 8.00.1, 2001.

11. (a) Lee, C.; Yang, W.; Parr, R. G. Phys. Rev. B 1988, 37, 785. (b) Becke, A. D. J. Chem. Phys. 1992, 96, 2155. (c) Becke, A. D. J. Chem. Phys. 1992, 97, 9173. (d) Becke, A. D. J. Chem. Phys. 1993, 98, 5648.

12. (a) Ditchfield, R.; Hehre, W. J.; Pople, J. A. J. Chem. Phys. 1971, 54, 724. (b) Hehre, W. J.; Ditchfield, R.; Pople, J. A. J. Chem. Phys. 1972, 56, 2257. (c) Hariharan, P. C.; Pople, J. A. Theor. Chim. Acta 1973, 28, 213.

13. (a) Ditchfield, R. Mol. Phys. 1974, 27, 789. (b) Wolinski, K.; Hilton, J. F.; Pulay, P. J. Am. Chem. Soc. 1990, 112, 8251. (c) Cheeseman, J. R.; Trucks, G. W.; Keith, T. A.; Frisch, M. J. J. Chem Phys. 1996, 104, 5497.

14. Helgaker, T.; Watson, M.; Handy, N. C. J. Chem. Phys. 2000, 113, 9402. (b) Sychrovsky, V.; Grafenstein, J.; Cremer, D. J. Chem. Phys. 2000, 113, 3530. (c) Barone, V.; Peralta, J. E.; Contreras, R. H.; Snyder, J. P. J. Phys. Chem. A 2002, 106, 5607.

15. Frisch, M. J.; Trucks, G. W.; Schlegel, H. B.; Scuseria, G. E.; Robb, M. A.; Cheeseman, J. R.; Montgomery, Jr., J. A.; Vreve, T.; Kudin, K. N.; Burant, J. C; Millam, J. M.; Iyengar, S. 
S.; Tomasi, J.; Barone, V.; Mennucci, B.; Cossi, M.; Scalmani, G.; Rega, N.; Petersson, G. A.; Nakatsuji, H.; Hada, M.; Ehara, M.; Toyota, K.; Fukuda, R.; Hasegawa, J.; Ishida, M.; Nakajima, T.; Honda, Y.; Kitao, O.; Nakai, H.; Klene, M.; Li, X.; Knox, J. E.; Hratchian, H. P.; Cross, J. B.; Adamo, C.; Jaramillo, J.; Gomperts, R.; Stratmann, R. E.; Yazyev, O.; Austin, A. J.; Cammi, R.; Pomelli, C.; Ochterski, J.; Ayala, P. Y.; Morokuma, K.; Voth, G. A.; Salvador, P.; Dannenberg, J. J.; Zakrzewski, V. G.; Dapprich, S.; Daniels, A. D.; Strain, M. C.; Farkas, O.; Malick, D. K.; Rabuck, A. D.; Raghavachari, K.; Foresman, J. B.; Ortiz, J. V.; Cui, Q.; Baboul, A. G.; Clifford, S.; Cioslowski, J.; Stefanov, B. B.; Liu, G.; Liashenko, A.; Piskorz, P.; Komaromi, I.; Martin, R. L.; Fox, D. J.; Keith, T.; Al-Laham, M. A.; Peng, C. Y.; Nanayakkara, A.; Challacombe, M.; Gill, P. M. W.; Johnson, B.; Chen, W.; Wong, M. W.; Gonzalez, C.; Pople J. A. GAUSSIAN 03, Rev. B.02, Gaussian, Inc., Pittsburgh PA, 2003. 